\title{
Nonalcoholic steatohepatitis critically rewires the ischemia/ reperfusion-induced dysregulation of cardiolipins and sphingolipids in mice
}

\author{
Sheng Yu ${ }^{1 \#}$, Kai Wang ${ }^{1 \#}$, Qingping Li $^{1 \#}$, Yiran Wei ${ }^{2 \#}$, Yiyi Li ${ }^{3}$, Qifan Zhang ${ }^{1}$, Pengxiang Huang ${ }^{1}$, \\ Hanbiao Liang ${ }^{1}$, Hang Sun ${ }^{1}$, Hongxian Peng ${ }^{1}$, Xixin Huang ${ }^{2}$, Cuiting Liu ${ }^{4}$, Jie Zhou ${ }^{1}$, Jianping Qian ${ }^{1}$, \\ Chuanjiang $\mathrm{Li}^{1}$
}

${ }^{1}$ Division of Hepatobiliopancreatic Surgery, Department of General Surgery, Nanfang Hospital, Southern Medical University, Guangzhou, China; ${ }^{2}$ The First Clinical College, Southern Medical University, Guangzhou, China; ${ }^{3}$ Department of Radiation Oncology, Nanfang Hospital, Southern Medical University, Guangzhou, China; ${ }^{4}$ Central Laboratory, Southern Medical University, Guangzhou, China

Contributions: (I) Conception and design: J Zhou, C Li, J Qian, Y Li, K Wang, Q Zhang, P Huang, H Liang, H Sun, H Peng, C Liu; (II) Administrative support: None; (III) Provision of study materials or patients: None; (IV) Collection and assembly of data: None; (V) Data analysis and interpretation: S Yu, Y Wei, Q Li; (VI) Manuscript writing: All authors; (VII) Final approval of manuscript: All authors.

\#These authors contributed equally to this work.

Correspondence to: Jie Zhou, MD. Division of Hepatobiliopancreatic Surgery, Department of General Surgery, Nanfang Hospital, Southern Medical University, Guangzhou, China. Email: jacky@smu.edu.cn; Jianping Qian. Division of Hepatobiliopancreatic Surgery, Department of General Surgery, Nanfang Hospital, Southern Medical University, Guangzhou, China. Email: qjp1982@smu.edu.cn; Chuanjiang Li. Division of Hepatobiliopancreatic Surgery, Department of General Surgery, Nanfang Hospital, Southern Medical University, Guangzhou, China. Email: licj@smu.edu.cn.

Background: Lipid dysregulation plays a fundamental role in nonalcoholic steatohepatitis (NASH), which is an emerging critical risk factor that aggravates hepatic ischemia/reperfusion (I/R) injury. However, the specific lipids that mediate the aggressive I/R injury in NASH livers have not yet been identified.

Methods: The mouse model of hepatic I/R injury on NASH was established on C56B/6J mice by first feeding the mice with a Western-style diet to induce NASH, then the NASH mice were subjected to surgical procedures to induce hepatic I/R injury. Untargeted lipidomics were performed to determine hepatic lipids in NASH livers with I/R injury through ultra-high performance liquid chromatography coupled with mass spectrometry. The pathology associated with the dysregulated lipids was examined.

Results: Lipidomics analyses identified cardiolipins (CL) and sphingolipids (SL), including ceramides (CER), glycosphingolipids, sphingosines, and sphingomyelins, as the most relevant lipid classes that characterized the lipid dysregulation in NASH livers with I/R injury. CER were increased in normal livers with I/R injury, and the I/R-induced increase of CER was further augmented in NASH livers. Metabolic pathway analysis revealed that the enzymes involved in the synthesis and degradation of CER were highly upregulated in NASH livers with I/R injury, including serine palmitoyltransferase 3 (Sptlc3), ceramide synthase 2 (Cers2), neutral sphingomyelinase 2 (Smpd3), and glucosylceramidase beta 2 (Gba2) that produced CER, and alkaline ceramidase 2 (Acer2), alkaline ceramidase 3 (Acer3), sphingosine kinase 1 (Sphk1), sphingosine-1-phosphate lyase (Sgpl1), and sphingosine-1-phosphate phosphatase 1 (Sgpp1) that catalyzed the degradation of CER. CL were not affected by I/R challenge in normal livers, but CL was dramatically reduced in NASH livers with I/R injury. Consistently, metabolic pathway analyses revealed that the enzymes catalyzing the generation of CL were downregulated in NASH-I/R injury, including cardiolipin synthase (Crls1) and tafazzin (Taz). Notably, the I/R-induced oxidative stress and cell death were found to be aggravated in NASH livers, which were possibly mediated by the reduction of CL and accumulation of CER. Conclusions: The I/R-induced dysregulation of CL and SL were critically rewired by NASH, which might potentially mediate the aggressive I/R injury in NASH livers.

Keywords: Nonalcoholic steatohepatitis (NASH); ischemia/reperfusion injury; lipidomics; lipid metabolism 
Submitted Mar 28, 2021. Accepted for publication Aug 26, 2021. Published online Nov 09, 2021.

doi: $10.21037 / \mathrm{hbsn}-21-133$

View this article at: https://dx.doi.org/10.21037/hbsn-21-133

\section{Introduction}

Nonalcoholic fatty liver disease (NAFLD) is a worldwide burgeoning health problem, which is predicted to be the primary cause of chronic liver diseases in the coming decades (1). NAFLD consists of a comprehensive disease spectrum of histology, which is categorized into nonalcoholic fatty liver (NAFL), nonalcoholic steatohepatitis (NASH), and cirrhosis. Among these, $\mathrm{NASH}$ is the progressive stage of NAFLD characterized by the coexistence of hepatocellular damage, inflammation, and fibrosis (2). Ischemia/reperfusion (I/R) injury is a typical pathophysiological process inducing liver injury and liver dysfunction (3). Emerging evidences demonstrate that NASH sensitizes livers to I/R injury by exaggerating hepatocellular damages and inflammation, resulting in advanced liver dysfunction (4). However, due to the incomplete understanding of the mechanism by which NASH aggravates hepatic I/R injury, an effective therapy for relieving hepatic I/R injury in NASH patients is still lacking.

Lipid dysregulation has been implicated to play fundamental roles in mediating the pathogenesis and progression of NASH $(3,5)$. Interestingly, certain lipids are also involved in hepatic I/ $\mathrm{R}$ injury by mediating hepatocellular damage, oxidative stress, and inflammation in normal livers $(6,7)$, such as glycerophospholipid (GSL) were known to have anti-oxidative activities (8). Ceramides (CER) with pro-apoptotic and pro-inflammatory activities were recently found to be increased in either NASH livers or normal livers with I/R injury $(6,9)$. Particularly, CER were implicated in mediating several signaling pathways in promoting hepatic injury linked to the progression of NASH or hepatic I/R injury (10-12). Our previous studies also identified unsaturated-long-chain CER and the CER metabolite sphingosine-1-phosphate as critical lipid mediators by regulating oxidative stress in NASH and NAFL with hepatic I/R injury, respectively $(13,14)$. Cardiolipins (CL) were reported to alleviate both oxidative stress and apoptosis $(15,16)$. Martens et al. demonstrated that I/R injury significantly reduced CL content and increased oxidized CL in livers, and the dysregulated CL might impair mitochondrial function by inducing oxidative stress (17). Together, these previous studies collectively suggest that dysregulation in lipids critically regulates liver injuries caused by NASH or hepatic I/R. However, much remains unclear about lipid dysregulations in mediating the aggressive I/R injury in NASH livers.

In this study, the dysregulation of lipid metabolism in NASH livers with I/R injury was investigated. By untargeted lipidomics analyses, our data illustrated an overview of dysregulated lipid spices in NASH livers with I/ $\mathrm{R}$ injury in mice. Among the detected lipids, sphingolipids (SL) and CL were identified as the most relevant lipid classes that characterized the lipid dysregulation in NASH livers with I/R injury. Moreover, the dysregulation of SL and CL potentially mediated the aggressive I/R injury in NASH livers. Targeting the dysregulation of SL and CL metabolism might be a therapeutic approach for aggressive I/R injury in NASH livers. We present the following article in accordance with the ARRIVE reporting checklist (available at https://hbsn.amegroups.com/article/ view/10.21037/hbsn-21-133/rc).

\section{Methods}

\section{Mouse model of bepatic I/R injury on NASH}

Six-week-old male C57B/6J mice were purchased from the animal experiment center of Southern Medical University (Guangzhou, China). Mice were fed with a Western pattern diet (TD.88137, Harlan; Southern Easton, MA, USA) for 10 weeks to establish NASH model (18), the critical dietary features of TD.88137 included: cholesterol $(0.2 \%$ total cholesterol), total fat (21\% by weight; $42 \% \mathrm{kcal}$ from fat), high in saturated fatty acids ( $>60 \%$ of total fatty acids) and high sucrose (34\% by weight). The normal mice were fed with a 60 Co sterilized maintenance feed produced by Beijing Huafukang Biological Technology Co. Ltd (Beijing, China, Cat. No. 1025). The information on the makeup of the diets was listed in Tables S1,S2. To induce the hepatic I/R injury, previously described procedures were followed (19). Briefly, under isoflurane inhalant anesthesia, 

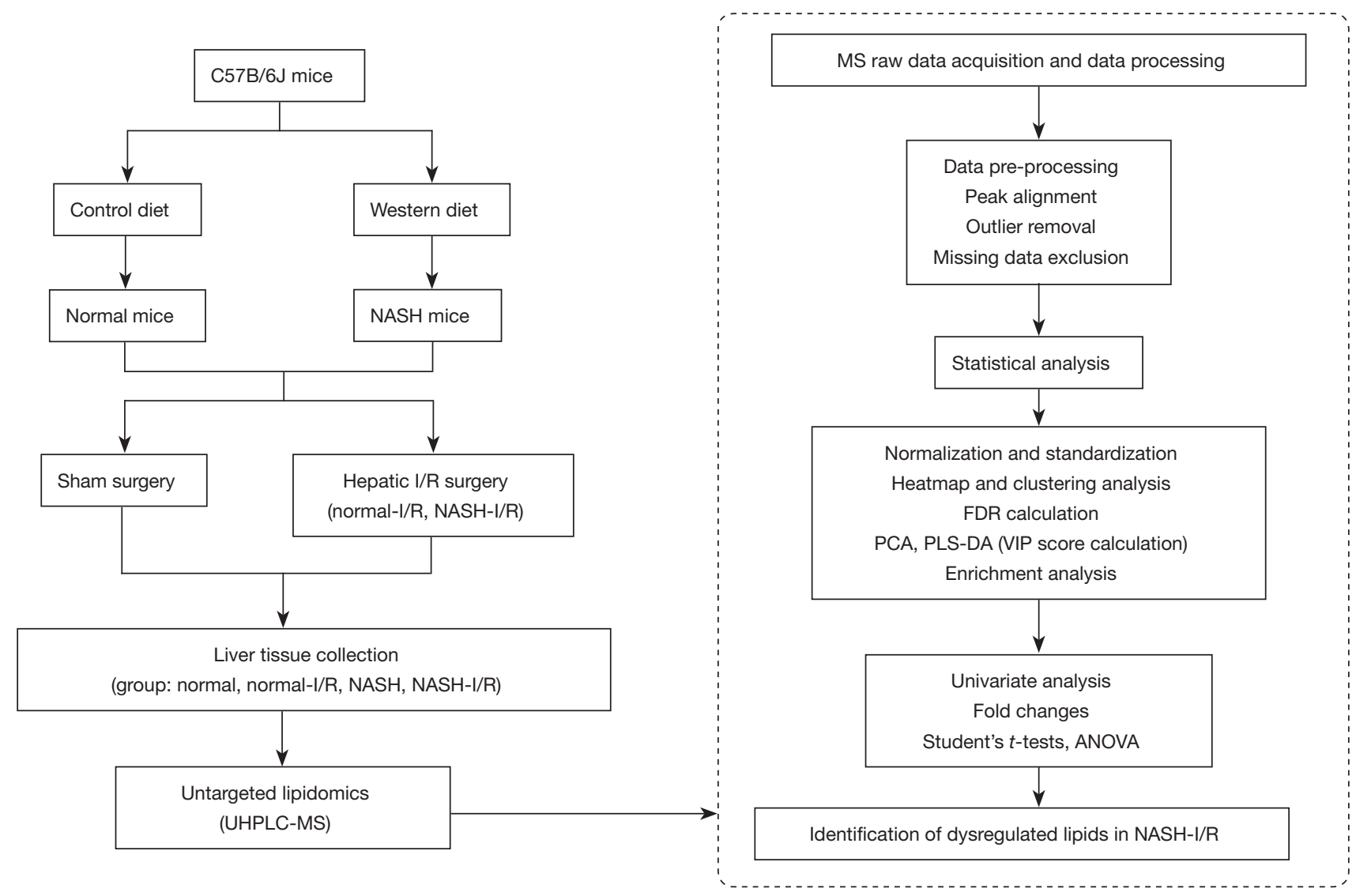

Figure 1 The workflow of experimental design and lipidomic analysis. NASH, nonalcoholic steatohepatitis; I/R, ischemia/reperfusion; UHPLC, ultra-high-performance liquid chromatography; MS, mass spectrometry; FDR, false discovery rate; PCA, principal component analysis; PLS-DA, projections to latent structures-discriminant analysis; VIP, variable importance in projection; ANOVA, analysis of variance.

an atraumatic vascular clamp was applied to produce $70 \%$ hepatic ischemia for a period of 60 minutes. Serum and liver tissues were collected at 24 hours after liver I/ $\mathrm{R}$ injury. The histology of liver I/ $\mathrm{R}$ injury was examined by measuring the necrosis area using Image $\mathrm{J}$ software (NCBI; Bethesda, MD, USA), necrosis was distinguished by disintegration of hepatic cords, hemorrhage, and neutrophil infiltration (20). A workflow of experimental design and lipidomic analysis was shown in Figure 1. Experiments were performed under a project license (No. NFYY-2019-028) granted by the institutional ethics committee of Nanfang Hospital Southern Medical University, in compliance with institutional guidelines for the care and use of animals.

\section{Histologic examination of $\mathrm{NASH}$}

The histology of NASH was evaluated according to steatosis areas, inflammatory foci numbers, and hepatic fibrosis score as previous study $(13,21)$. Liver tissues embedded in paraffin blocks were sectioned and tissue sections were stained with hematoxylin and eosin ( $\mathrm{H} \& \mathrm{E})$ for histologic analysis. Inflammatory foci were counted in $\times 20$ field of views, five random fields were scored for each section. Sirius red-stained sections were used to score fibrosis as previously described (21). Briefly, no fibrosis was scored 0 , perisinusoidal or periportal fibrosis 1 , perisinusoidal and portal/periportal fibrosis 2, bridging fibrosis 3 , and cirrhosis, widespread fibrosis, and hepatocyte nodule formation 4 . Five random fields were scored for each section. Stained sections were used for scoring under Intelligently Designed Microscope (Olympus; Shinjuku-ku, Tokyo, Japan) in a blind manner.

\section{Quantitative real-time polymerase chain reaction (PCR)}

Total RNA was isolated using RNeasy Mini Kit (Qiagen) 
according to the manufacturer's instructions. mRNA was reversely transcribed to cDNA by $5 \times$ PrimeScript RT Master Mix (TaKaRa; Kusatsu, Shiga, Japan). Quantitative polymerase chain reaction (qPCR) analyses were done on an ABI Prism 7000 sequence detection system (Thermo; Ringoes, NJ, USA). Quantitation of specific gene expression was calculated using the delta-delta Ct method. $\beta$-Actin was used as the reference gene. Primer sequences were shown in Table S3.

\section{Oil Red O staining}

Frozen liver tissue sections were prepared, Oil Red O staining was performed as described (22) with minor modifications. Briefly, fresh liver tissues from mice were embedded in Tissue-Tek Optimal Cutting Temperature (OCT) compound and sectioned. Sections were incubated with Oil Red O solution ( $0.375 \%$, wt/vol) (Sigma-Aldrich; St. Louis, MO, USA) for 5 minutes. Sections were examined under Intelligently Designed Microscope (Olympus; Shinjuku-ku, Tokyo, Japan).

\section{Histopathology and immunobistochemistry}

After deparaffinized, liver sections were stained with $\mathrm{H} \& \mathrm{E}$ according to the manufacturer's instructions. Liver sections were subjected to immunostaining with 4 hydroxynonenal (4 HNE) (Abcam; Cambridge, USA, ab46545) and lymphocyte antigen 6G (Ly6G) antibody (Abcam; Cambridge, USA, ab238132) to examine oxidative stress and inflammatory infiltration, respectively. Terminal deoxynucleotidyl transferase dUTP nick end labeling (TUNEL) staining was performed using a TACS-XL In Situ Apoptosis Detection Diaminobenzidine (DAB) Kit (Trevigen; Gaithersburg, USA) according to the manufacturer's instructions. Sections were examined under Intelligently Designed Microscope (Olympus; Shinjuku-ku, Tokyo, Japan).

\section{Western blotting}

Liver tissues were lysed in ice-cold RIPA lysis buffer (Solarbio, Beijing, China). Equal protein lysates were separated by $10 \%$ SDS-PAGE and then transferred to polyvinylidene fluoride (PVDF) membrane. After blocking with $5 \%$ BSA, the membranes were incubated with antibodies against $4 \mathrm{HNE}$ (Abcam; Cambridge, USA, ab46545), cleaved caspase-3 (Cell Signaling Technology; Danvers, USA, 9664), cleaved-PARP (Cell
Signaling Technology; Danvers, USA, 5625S), respectively. HRP-conjugated secondary antibodies (Cell Signaling Technology; Danvers, USA, 7074) were applied. The signals were visualized using the efficient chemiluminescence (ECL) detection kit (GE Healthcare, Little Chalfont, UK). $\beta$-Actin (Bioworld; Minneapolis, USA, AP0060) was used as the internal control.

\section{Untargeted lipidomics and analyses}

Lipid extraction from the liver tissues was performed followed the Bligh and Dyer procedure as described (23). The samples were analyzed by a Thermo Fisher Scientific Vanquish Flex ultra-high-performance liquid chromatography (UHPLC) equipped with Thermo Fisher Scientific Orbitrap Fusion Tribrid High-Resolution Mass Spectrometer (Thermo Fisher Scientific, Waltham, MA, USA). The identification of lipid molecular species was performed using Lipid Search software (Thermo Fisher Scientific; Waltham, MA, USA). These lipid-profiling data were subjected to further analyses using the online Metaboanalyst tool (www.metaboanalyst.ca). The data first underwent a normalization using values of quality control samples. Missing values for lipids that were not detected in all samples were excluded. Log2 transformation was conducted to create a heatmap. Heatmap analysis with clustering was performed using Euclidean distance measuring and a Ward clustering algorithm. The individual lipid species that were critically altered by I/R, NASH, or NASH livers with I/R injury were screened according to the false discovery rate (FDR) by using Benjamini and Hochberg adjustment. Log10-transformed data were autoscaled for principal component analysis (PCA) and projections to latent structures discriminant analysis (PLSDA). The contribution of lipid classes to the PLS-DA model was evaluated by variable importance in projection (VIP) score. The lipid classes with VIP score $>1.0$ and $\mathrm{P}$ value $<0.05$ were considered as the most critical lipids that were affected by I/R, NASH, or NASH livers with I/ $\mathrm{R}$ injury. The differences of the selected lipid species and lipid classes were then analyzed by using one-way analysis of variance (ANOVA) followed by Tukey's post-hoc test or Student's $t$-test depending on the number of groups that were compared. According to the results of ANOVA or Student's $t$-test, the lipids that were critically affected by NASH livers with I/R injury were eventually identified. Metabolic pathway analysis of CL was performed using an online BioPAN tool (lipidmaps.org/biopan) (24). 


\section{Statistical analyses}

Statistical analyses were performed using the Student's $t$-test or AVONA using IBM SPSS Statistics for Windows version 22.0 (IBM; Armonk, NY, USA). P values $<0.05$ were considered significant.

\section{Results}

\section{Hepatic I/R injury is aggravated in NASH mice}

To facilitate the investigation on lipid dysregulation in NASH livers with I/R injury, a murine model of NASH through feeding mice with a saturated-fatty-acid-enriched Western pattern diet was established (18). During induction of NASH, mouse bodyweights (Figure $2 A$ ) and liver weight were significantly increased (Figure $2 B$ ). Steatohepatitis was successfully induced in the NASH mice, indicating by elevation of alanine aminotransferase (ALT) and aspartate aminotransferase (AST) (Figure 2C), upregulation of pro-inflammatory cytokines (Figure 2D), hepatic lipid accumulation (Figure $2 E, 2 F$ ), inflammatory infiltration (Figure $2 E, 2 G, 2 H$ ) and fibrous formation (Figure 2E, 2I). Next, NASH mice were subjected to the hepatic I/R challenge. Serum and liver tissues were collected at $24 \mathrm{~h}$ after liver I/R injury. We found that hepatocellular necrosis and inflammatory infiltration induced by hepatic I/R injury were exaggerated in NASH livers compared with that in normal liver (Figure 27,2K). Liver function test revealed that elevation of ALT, AST, and total bilirubin (TBIL) levels induced by hepatic I/ $\mathrm{R}$ challenge was significantly promoted by NASH (Figure $2 L-2 N$ ). Together, these data collectively demonstrated that hepatic I/R injury was substantially exaggerated in $\mathrm{NASH}$ mice.

\section{The I/R-induced lipid dysregulation in NASH livers is distinct from that in normal livers}

Dysregulation of lipid metabolism has been implicated in both NASH and hepatic I/R injury $(6,17,25)$, but much still remains unclear how NASH impacts the I/R-induced lipid dysregulation. To this end, we performed untargeted lipidomics to determine the potential alterations of hepatic lipids in NASH livers with I/R injury. Overall, a total of 1,578 individual lipid species, which belonged to 26 classes of hepatic lipids, were detected in mouse livers. Heatmap with clustering and PCA analysis demonstrated that the I/R-induced alteration of lipid content in NASH livers was distinct from that in normal livers (Figure $3 A-3 C$ ). According to the FDR values, DG (18:4/18:2), TG (4:0/18:2/18:2), TG (4:0/10:0/16:0), CL (23:0/18:1/20:4/20:4), TG (18:0p/18:1/18:2), CL (22:6/18:1/20:0/22:6), LPC (19:3), PC (16:1/16:1), CL (18:2/18:0/18:0/22:0), and TG (20:1/18:2/24:1) were identified as the top 10 lipid species that were significantly altered by NASH induction or by I/R injury (Figure $3 D$ ). Notably, TG (4:0/18:2/18:2), CL (23:0/18:1/20:4/20:4), TG (18:0p/18:1/18:2), CL (22:6/18:1/20:0/22:6), CL (18:2/18:0/18:0/22:0), TG (20:1/18:2/24:1, LPC(19:3), and PC (16:1/16:1) were identified as the key lipid signatures of I/R injury in NASH livers. In detail, TG (4:0/18:2/18:2) was significantly upregulated in normal livers with $\mathrm{I} /$ $\mathrm{R}$ injury but decreased in NASH livers with $\mathrm{I} / \mathrm{R}$ injury (Figure 3D). CL $(23: 0 / 18: 1 / 20: 4 / 20: 4)$, TG (18:0p/18:1/18:2), CL $(22: 6 / 18: 1 / 20: 0 / 22: 6)$, CL (18:2/18:0/18:0/22:0), and TG $(20: 1 / 18: 2 / 24: 1)$ were significantly decreased by I/R injury in normal livers, and the I/R-induced decrease of these lipids was further enhanced in NASH livers (Figure 3D). LPC (19:3) and PC (16:1/16:1) were also significantly decreased by I/R injury, but they were maintained at higher levels in NASH livers (Figure 3D). These data suggested that the lipid dysregulation in NASH livers with I/R injury was distinct from that in the normal liver with I/R injury.

\section{Lipid classes of SL and CL are critically altered in NASH livers with I/R injury}

The dysregulation of lipid classes in NASH livers with I/ $\mathrm{R}$ injury was further investigated. In total, 26 classes of hepatic lipids were detected. PCA analysis demonstrated that NASH and I/R injury substantially altered lipid classes in livers, and the I/R-induced alteration of lipid classes in NASH livers was different from that in normal livers (Figure 4A,4B). CER, triglycerides (TG), CL, sphingosines (SPH), GSL, lysophosphatidylethanols (LPEt), phosphatidylinositol phosphate (PIP), sphingomyelins (SM), and lysophosphatidylinositol (LPI) were identified as the relevant lipid classes that were altered by NASH induction or by I/R injury (Figure 4C). Notably, CER, SPH, GSL, and SM which belonged to SL family were largely dysregulated in NASH livers with I/R injury (Figure 4D). GSL and CER were slightly but significantly increased in normal livers with I/R injury, but SM and SPH were not affected (Figure $4 D$ ). Compared with the dysregulation of SL in normal livers with I/R injury, GSL and SM were 

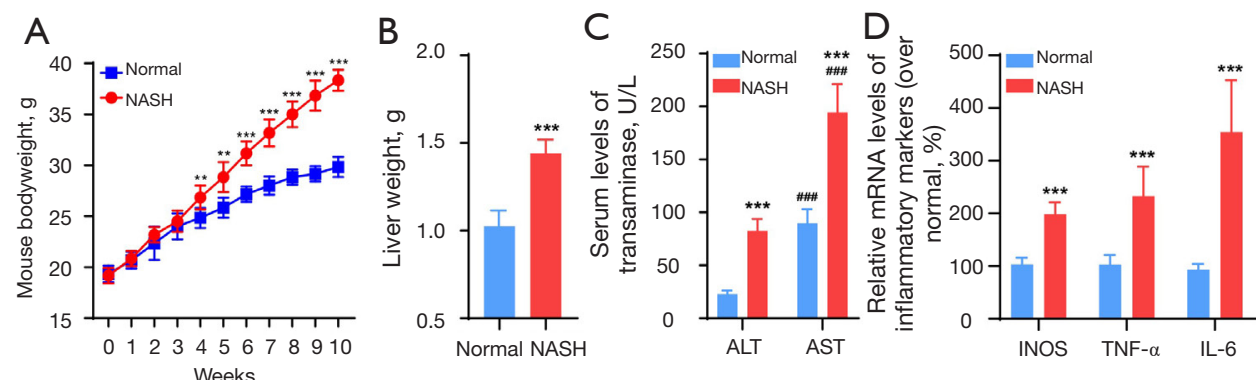

$\mathrm{E}$

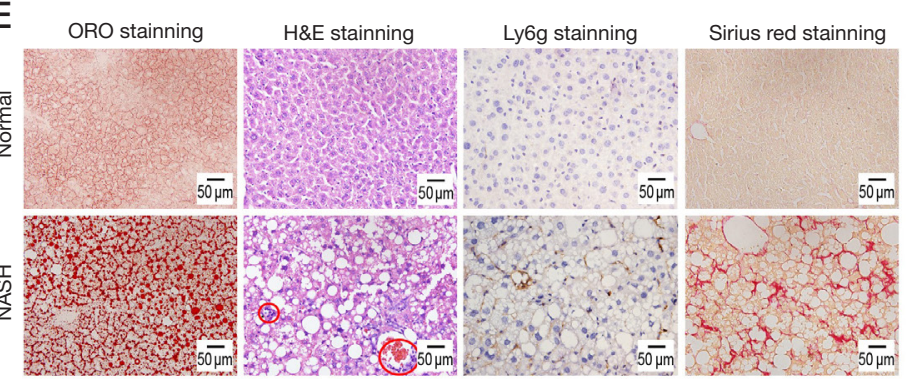

F

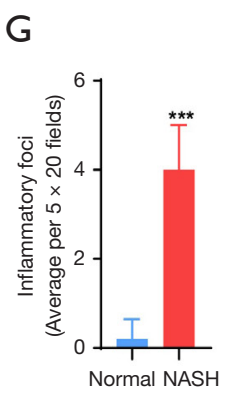

$\mathrm{H}$

I

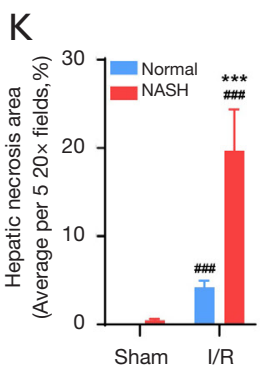

$\mathrm{L}$
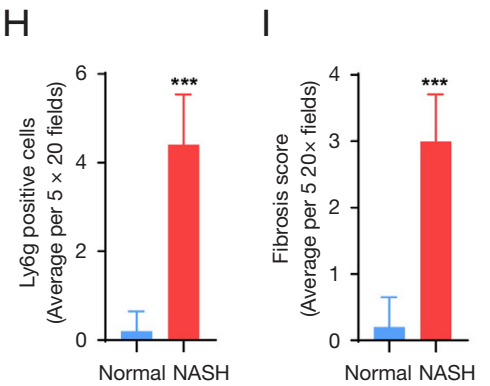

$\int$ Normal-sham
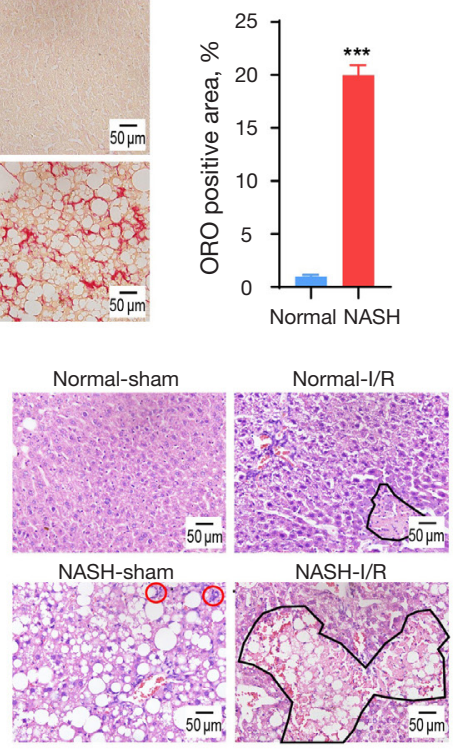

$M$
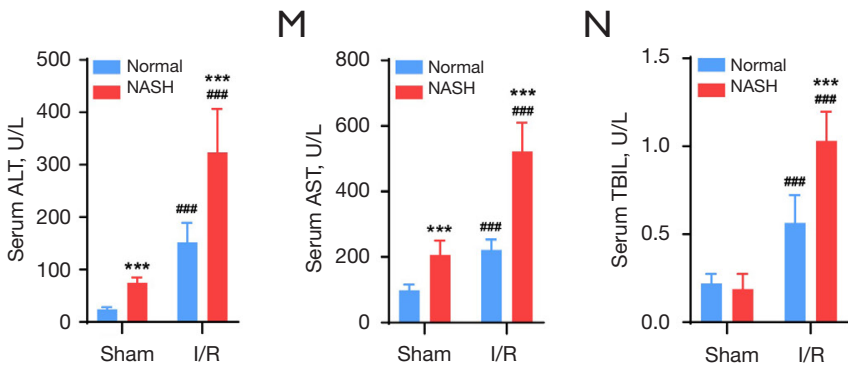

Figure 2 NASH exaggerates hepatic I/R injury in mice. C57BL/6J Mice were treated with a Western pattern diet for up to 10 weeks to induce NASH, and then hepatic I/R injury was induced by 1-hour ischemia followed by 24-hour reperfusion. (A) Mouse bodyweight changes were recorded once per week. (B) Mouse liver weights were recorded at 10 weeks after HFD treatment. (C) The serum levels of ALT and AST in NASH livers and normal livers were measured. (D) Inflammation related genes, including INOS, TNF-a, IL-6 were measured by qPCR assays. (E) Liver sections were subjected to ORO staining, H\&E staining, Ly6g immunostaining, and Sirius red staining. ORO staining on liver sections was performed for evaluating steatosis. Ly6g staining was performed to stain the lymphocytes in the liver sections. Sirius red staining was performed for evaluating fibrosis formation. (F) The staining density of ORO was quantified by Image J software. (G) Hepatic inflammatory foci that were marked by red cycle were counted to evaluate inflammatory infiltration. (H) Ly6g positive cells were quantified as mentioned in Methods. (I) Fibrosis was scored as described in "Methods" section. (J,K) Liver sections of normal mice and NASH mice with or without I/R injury were subjected to H\&E staining, and the red circle indicate the inflammatory cell infiltrations, the dot line area indicates area of necrosis (D). The area of necrosis was quantified using Image J software (K). (L-N) The level of serum ALT, AST, and TBIL were measured

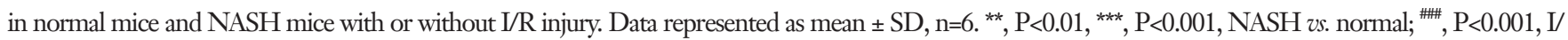
$\mathrm{R}$ vs. sham. NASH, nonalcoholic steatohepatitis; ALT, alanine aminotransferase; AST, aspartate aminotransferase; ORO, Oil Red O; H\&E, hematoxylin and eosin; I/R, ischemia/reperfusion; qPCR, quantitative polymerase chain reaction; TBIL, total bilirubin; HFD, high-fat diet; SD, standard deviation. 
A

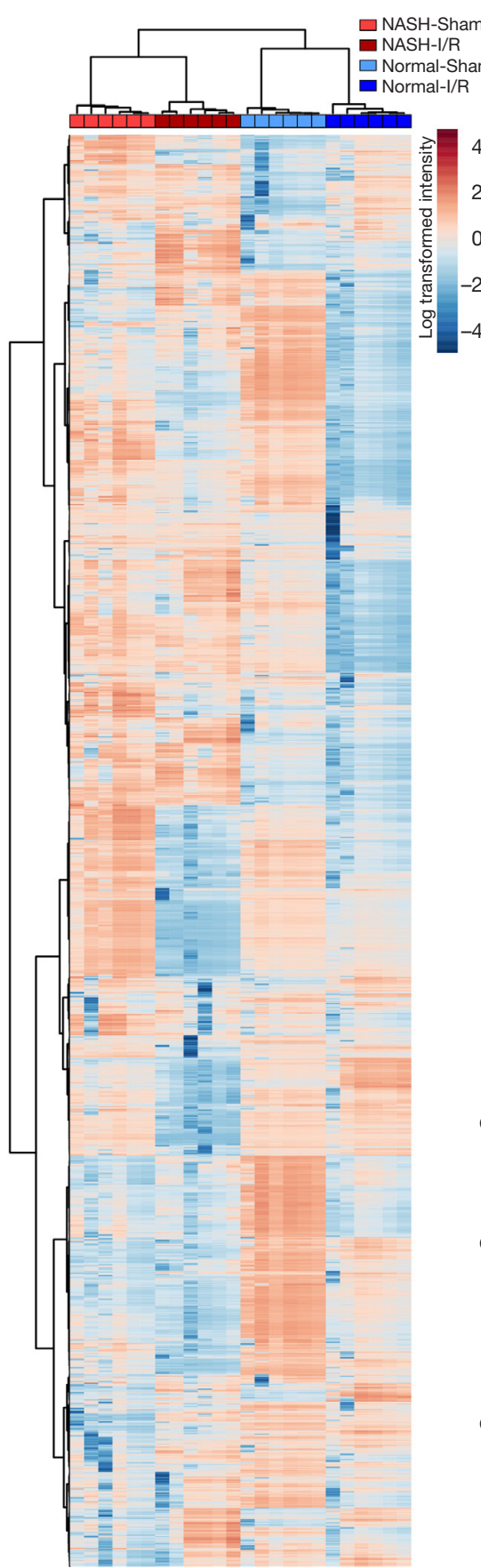

B
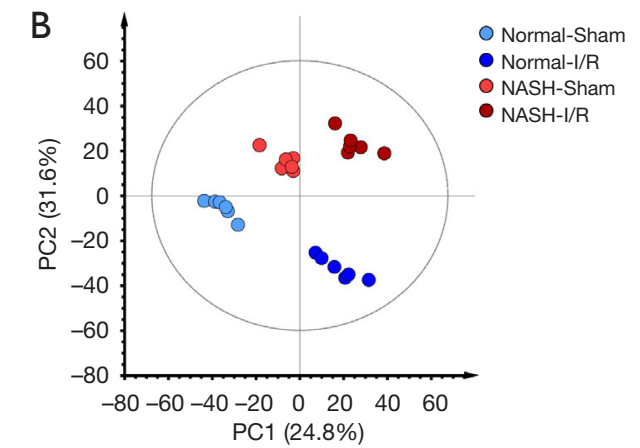

C
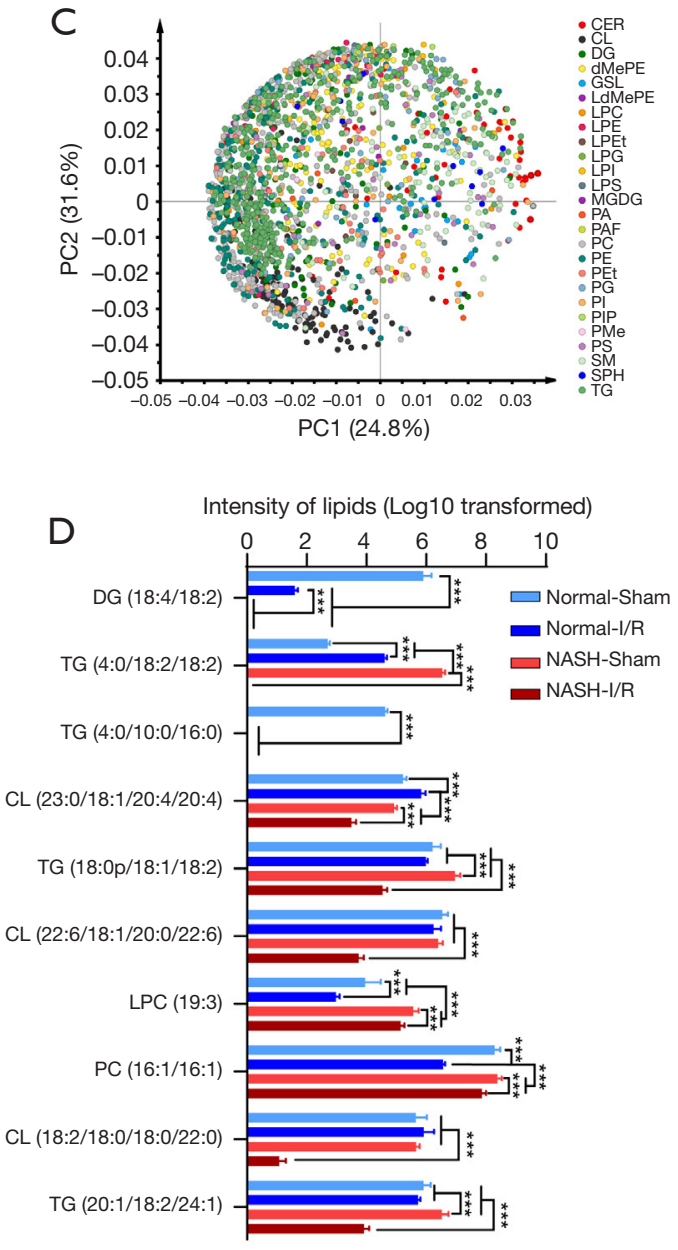

Figure 3 The I/R-induced lipid dysregulation in NASH livers is distinct from that in normal livers. Mouse liver samples were collected from normal mice and NASH mice with or without I/R injury. After lipid extraction, untargeted lipidomics analyses were performed by UHPLC-MS. In total, 26 classes of lipids, including 1,578 individual lipid species, were analyzed. (A) Heatmap with clustering analysis was performed for the 1,578 individual lipid species. (B,C) PCA analysis demonstrated that the differences in the lipid profile of 1,578 individual lipid species among the tested mice. (D) The individual lipids altered by NASH induction or by I/R injury were screened by FDR values. The lipids with FDR values ranking at the top 10 were illustrated. Data represented as mean $\pm \mathrm{SD}, \mathrm{n}=6 .{ }^{* * *}, \mathrm{P}<0.001$. NASH, nonalcoholic steatohepatitis; I/R, ischemia/reperfusion; UHPLC, ultra-high-performance liquid chromatography; MS, mass spectrometry; PCA, principal component analysis; FDR, false discovery rate; SD, standard deviation. 

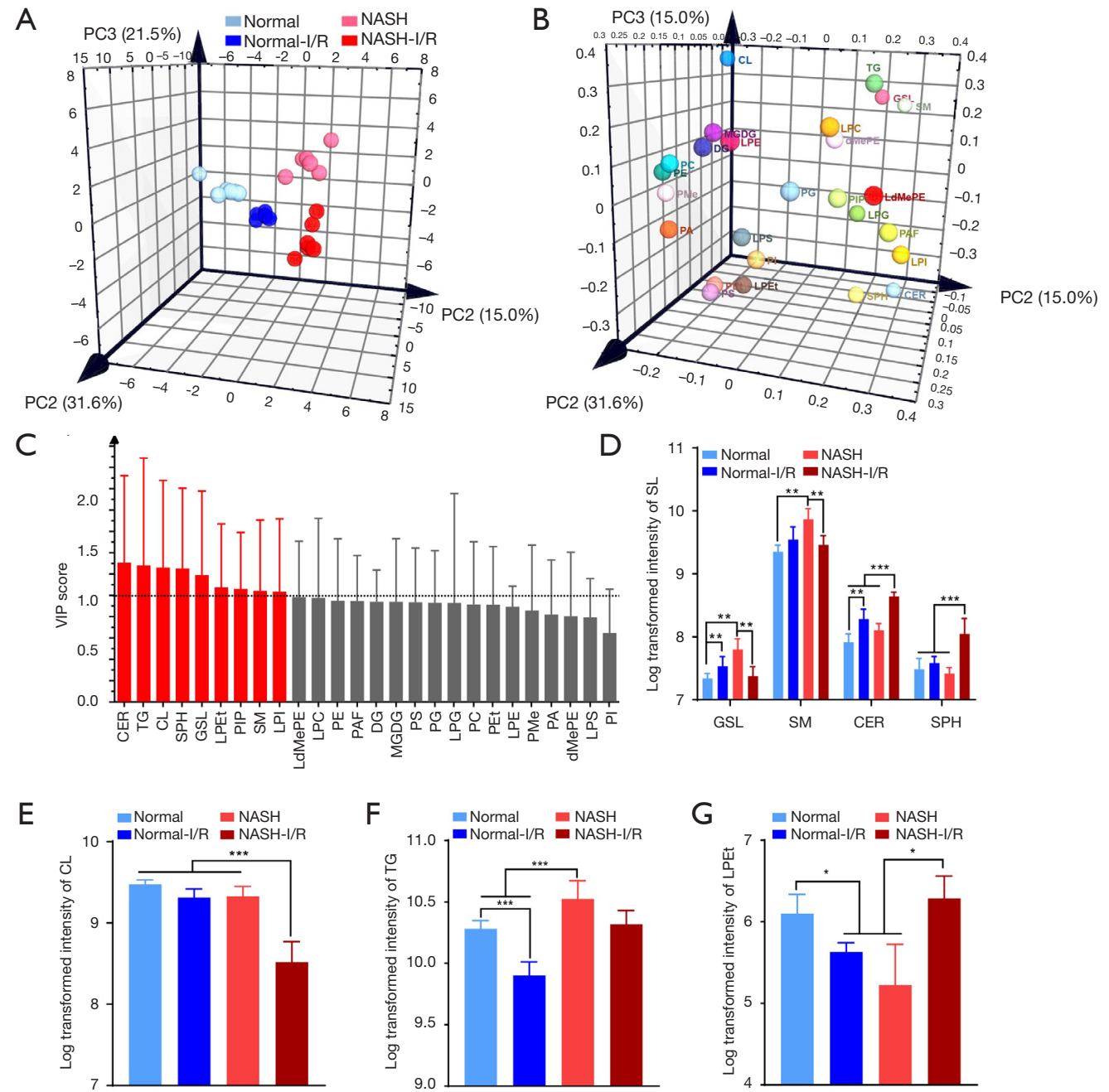

$\mathrm{H}$
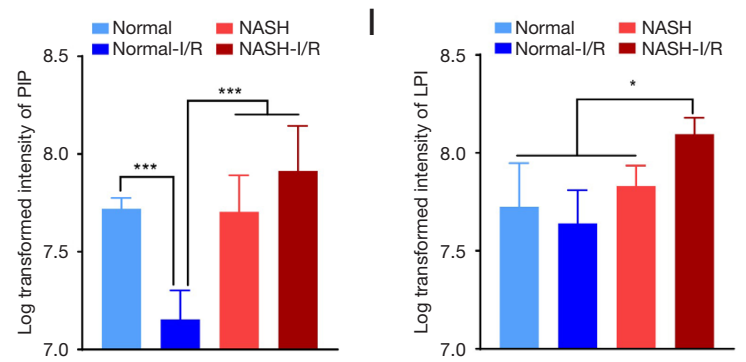

Figure 4 Lipid classes of SL and CL are critically dysregulated in NASH livers with I/R injury. Mouse liver samples were collected from normal mice and NASH mice with or without I/R injury. Untargeted lipidomics analyses were performed by UHPLC-MS. The detected lipid classes were analyzed. (A,B) PCA analysis demonstrated that the differences in the lipid profile of 26 lipid classes among the tested mice. (C) VIP scores were obtained from the PLS-DA analysis for 26 lipid classes. (D) The intensity of SL, including GSL, SM, CER, and SPH. (E) The intensity of CL. (F) The intensity of TG. (G) The intensity of LPEt. (H) The intensity of PIP. (I) The intensity of LPI. Data represented as mean $\pm \mathrm{SD}, \mathrm{n}=6 .{ }^{*}, \mathrm{P}<0.05,{ }^{* *}, \mathrm{P}<0.01,{ }^{* *}, \mathrm{P}<0.001$. NASH, nonalcoholic steatohepatitis; I/R, ischemia/ reperfusion; SL, sphingolipids; CL, cardiolipins; UHPLC, ultra-high-performance liquid chromatography; MS, mass spectrometry; PCA, principal component analysis; VIP, variable importance in projection; PLS-DA, projections to latent structures discriminant analysis; GSL, glycerophospholipid; SM, sphingomyelins; CER, ceramides; SPH, sphingosines; TG, triglycerides; LPEt, lysophosphatidylethanols; PIP, phosphatidylinositol phosphate; LPI, lysophosphatidylinositol; SD, standard deviation. 
significantly decreased, while CER and SPH were increased to higher levels in NASH livers with I/R injury (Figure 4D). In addition to SL, CL were substantially decreased in NASH livers with I/R injury, which were not altered in normal livers with $\mathrm{I} / \mathrm{R}$ injury (Figure $4 E$ ). TG were found to be increased in NASH livers without change upon I/R injury (Figure 4F). LPEt was decreased in normal livers with I/R injury but increased in NASL livers with I/R injury (Figure $4 G$ ). PIP was decreased in normal livers with I/R injury, but they were not significantly affected in NASL livers with I/R injury (Figure $4 H$ ). LPI was significantly increased in NASH livers with I/R injury (Figure $4 I$ ). Together, these data demonstrated that the SL, CL, LPEt, and LPI were the relevant lipid classes characterizing the lipid dysregulation in NASH livers with I/R injury. SL and CL were the most critical lipid classes being dysregulated in NASH livers with I/R injury.

\section{Generation and degradation of CER are bighly upregulated in NASH livers with I/R injury}

Dysregulation of SL metabolites were previously implicated in promoting NASH and hepatic I/R injury $(26,27)$. Since SL family was identified as the most relevant lipid classes that were dysregulated in NASH livers with I/R injury, the dysregulation in SL metabolic pathway in response to I/ $\mathrm{R}$ injury in NASH livers was further investigated. PCA analysis revealed that alteration of SL in response to I/ $\mathrm{R}$ injury in NASH livers was distinct from that in normal livers (Figure $5 A, 5 B$ ). Notably, SL was not substantially altered in normal livers upon I/R injury (Figure $5 A, 5 B$ ). $\mathrm{SPH}$ and CER were found as the major upregulated SL in NASH livers with I/R injury (Figure $5 B$ ). In order to further characterize the dysregulation in SL metabolic pathway in NASH livers with I/R injury, qPCR arrays were performed to measure the alteration in mRNA levels of SL metabolic enzymes (Figure 5C). We found that I/R challenge significantly upregulated serine palmitoyltransferase long chain base subunit 3 (Sptlc3) and ceramide synthase 2 (Cers2) that catalyzed CER de novo synthesis (Figure 5C). Neutral sphingomyelinase 3 (Smpd3) and glucosylceramidase 2 (Gba2), which respectively degrades SM and GSL to produce CER, were also upregulated by $\mathrm{I} / \mathrm{R}$ challenge in normal livers (Figure $5 \mathrm{C}$ ). Moreover, I/R challenge significantly upregulated alkaline ceramidase 2 (Acer2), alkaline ceramidase 3 (Acer3), sphingosine kinase 1 (Sphk1), sphingosine-1-phosphate lyase 1 (Sgpl1), and sphingosine-1-phosphate phosphatase 1 (Sgpp1) that catalyzed the degradation of CER (Figure 5C). Notably, NASH dramatically enhanced the I/R-induced upregulation of Cers2, Acer2, Sphk1, and Smpd3 (Figure 5C). Substrate specificity of SL enzymes has been implicated in controlling the levels of specific SL, such as Cers2 prefers long-chain fatty acids as subtracts to generate long-chain CER (28). Therefore, we analyzed the alteration in CER and SM with different lengths of fatty acyl chains. I/R injury was found to significantly increased the levels of saturated CER with 16 to 18 carbons of fatty acyl chains and unsaturated CER with 18 to 28 carbons of fatty acyl chains in normal livers (Figure 5D). However, in NASH livers, I/R injury distinctly increased the levels of saturated CER with 20 to 26 carbons of fatty acyl chains, which were not affected in normal livers (Figure 5D). SM were not significantly affected by I/ $\mathrm{R}$ injury in normal livers, but SM with 20 to 24 carbons of fatty acyl chains was highly increased by NASH induction and dramatically decreased upon I/R injury (Figure $5 E$ ). These data altogether indicated that NASH promoted the I/R-activated generation and degradation of CER to enhance CER turnover in livers, especially the saturated long-chain CER.

\section{Generation of $C L$ is inactivated in NASH livers with $I / R$ injury}

CL are a group of mitochondrial lipids with anti-oxidative activities, which were previously found to be dysregulated in either NASH or I/R-injured livers $(17,29)$. Since we found that the lipid class of CL was one of the most downregulated lipids in NASH livers with I/R injury, the alteration in CL metabolism in I/R-injured NASH livers was further investigated. A total of 64 species of CL were detected by untargeted lipidomics (Figure 6A). Heatmap with clustering and PCA analyses demonstrated that alteration in CL species in response to I/R challenge in NASH livers was distinct from that in normal livers (Figure 6A,6B). Notably, NASH induction per se did not generally alter the levels of CL (Figure $6 B$ ). In normal livers with I/R injury, CL (16:1/16:1/16:1/16:1) and CL (17:1/16:1/16:1/16:1) were found as the most upregulated CL, CL (18:1/16:0/18:1/20:0) was the most downregulated CL (Figure 6C). CL (18:2/18:0/18:0/22:0) was found as the most downregulated CL in NASH livers with I/R injury (Figure 6D). Enrichment analysis further revealed that I/R injury in normal livers activated cardiolipin synthase (Crls1), which synthesized CL using phosphatidylglycerols (PG) as substrates (Figure 6E), but NASH suppressed the CL generation in the condition of I/R injury by inhibiting Crls1 and tafazzin (Taz), which 

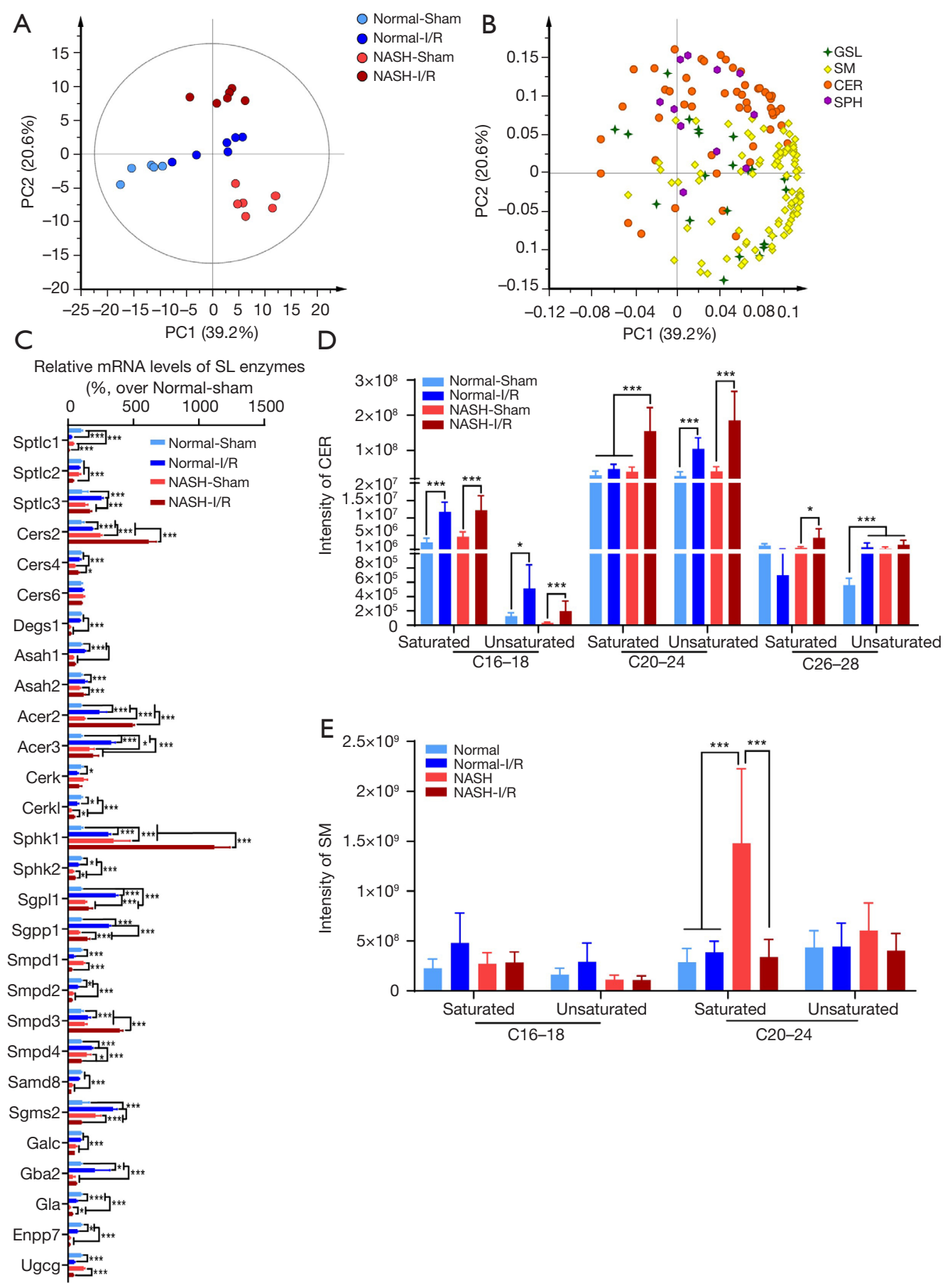

Figure 5 Alteration of SL metabolism in NASH livers with I/R injury. The detected SL, including GSL, SM, CER, and SPH were analyzed. (A,B) PCA analysis demonstrated that the differences of SL profile among the tested mice. (C) Total RNAs were extracted from the harvested samples of normal mice and NASH mice with or without I/R injury. The levels of mRNAs of SL metabolic enzymes were measured by qPCR assays. (D) The intensity of CER classified as saturated and unsaturated CER with different fatty acyl chains were analyzed. (E) The intensity of SM classified as saturated and unsaturated SM with different fatty acyl chains were analyzed. Data represented as mean $\pm \mathrm{SD}, \mathrm{n}=6 .{ }^{*}, \mathrm{P}<0.05 ;{ }^{* * *}, \mathrm{P}<0.001$. I/R, ischemia/reperfusion; NASH, nonalcoholic steatohepatitis; GSL, glycerophospholipid; SM, sphingomyelins; CER, ceramides; SPH, sphingosines; SL, sphingolipids; PCA, principal component analysis; qPCR, quantitative polymerase chain reaction; $\mathrm{SD}$, standard deviation. 
A

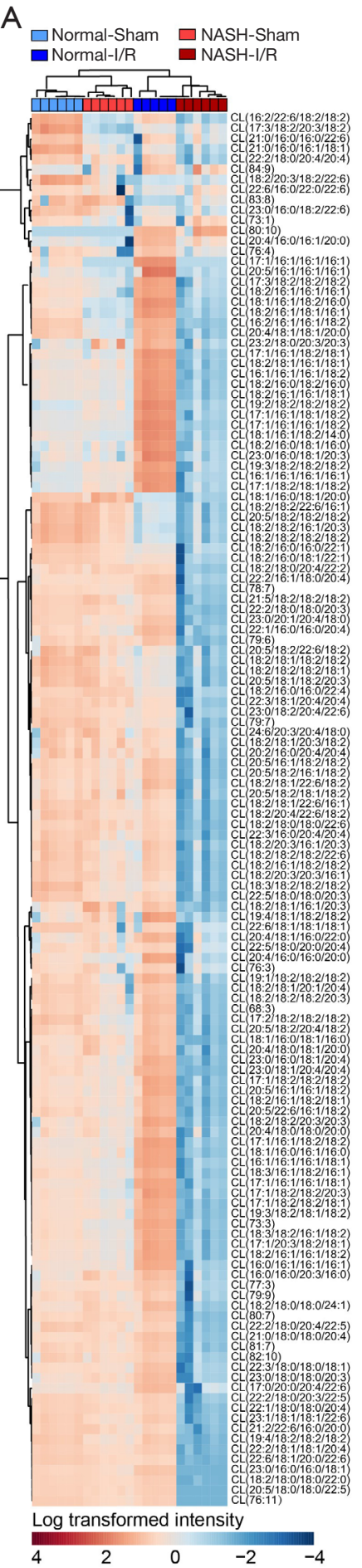

B

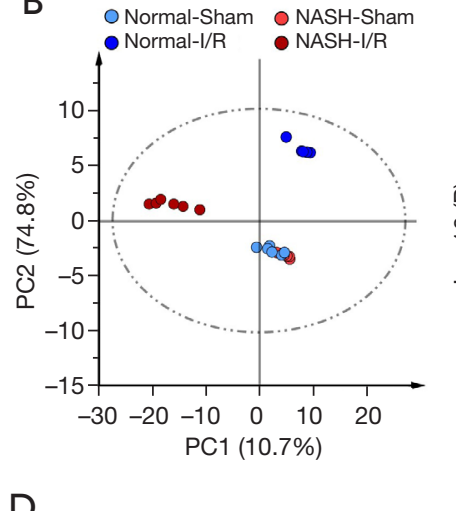

$\mathrm{D}_{10}$

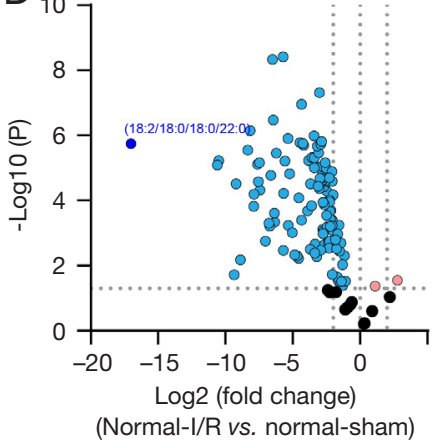

$\mathrm{F}$

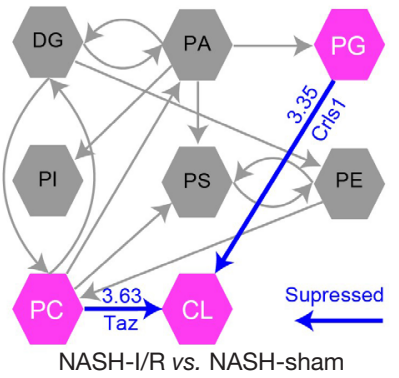

C

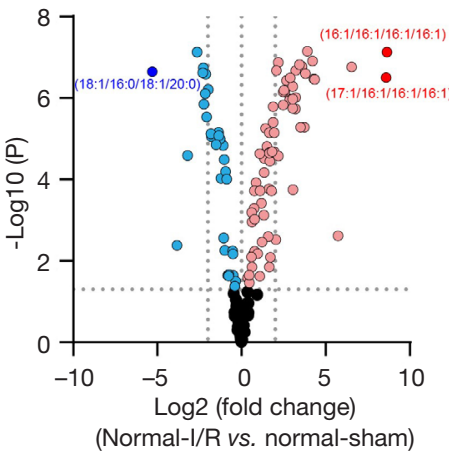

E

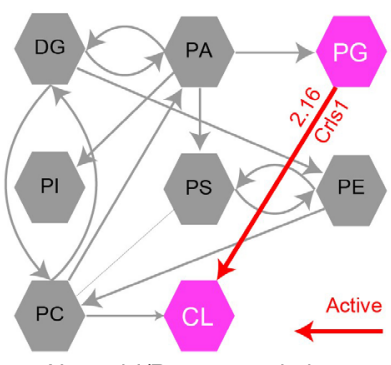

Normal-I/R vs. normal-sham

Figure 6 Dysregulation of CL metabolism in NASH livers with I/R injury. The detected CL were analyzed. (A) Heatmap with clustering analysis was performed for individual CL species. (B) PCA analysis demonstrated the differences in CL profile among the tested mice. (C) Volcano plot analysis demonstrated the alteration of $\mathrm{CL}$ in normal livers with or without $\mathrm{I} / \mathrm{R}$ injury. (D) Volcano plot analysis demonstrated the alteration of CL in NASH livers with or without I/R injury. (E) Enrichment analysis of CL metabolic pathway in normal livers with or without I/R injury. (F) Enrichment analysis of CL metabolic pathway in NASH livers with or without I/R injury. Data represented results from 6 individual mice of each group. NASH, nonalcoholic steatohepatitis; I/R, ischemia/reperfusion; CL, cardiolipins; PCA, principal component analysis. 
produced CL using PG and phosphatidylcholines (PC) as substrates, respectively (Figure $6 F$ ). Together, these findings suggested that NASH dramatically decreased the levels of CL species in the condition of I/R injury, mainly by inhibiting the synthesis of CL.

\section{The aggressive I/R injury in NASH livers is potentially mediated by the accumulation of CER and reduction of CL}

Accumulation of CER and decrease of CL have been shown to promote cell death, oxidative stress, and inflammation $(6,17,30,31)$. Since the accumulation of CER along with decreases of CL were found in NASH livers with I/R injury, the correlation between the aggressive hepatic injury and these dysregulated lipids was examined in terms of cell death and oxidative stress. TdT-mediated dUTP-biotin nick end labeling (TUNEL) assays demonstrated that the I/R-induced apoptosis was significantly exaggerated in NASH livers (Figure 7A,7B). Results of immune-blotting assays on quantitation of apoptotic markers also showed that protein levels of cleaved-caspase- 3 and cleaved-PARP were both upregulated to significantly higher levels in I/ R-injured NASH livers than that in normal livers with I/R injury (Figure 7C). Oxidative stress was assessed by evaluating expression levels of $4 \mathrm{HNE}$, which is one of the major products at the end step of lipid peroxidation (32). We found that the I/R-induced upregulation of $4 \mathrm{HNE}$ was significantly enhanced in NASH livers, indicating by more $4 \mathrm{HNE}$ positive cells and higher expression levels of 4 HNE in NASH livers with I/R injury (Figure $7 D-7 F$ ). Together, these findings collectively demonstrated that NASH aggravated hepatic I/R injury through augmenting cell death and oxidative stress, which could be potentially mediated by the accumulation of CER and decrease of CL.

\section{Discussion}

The understanding of the mechanism by which NASH exaggerates hepatic I/R injury has not yet been well elucidated. Previous studies have implicated the role of hepatic lipids in NASH or I/R injury $(6,17,26,27,29,33)$. This study explored the landscapes of dysregulated lipids in NASH livers with I/R injury in mice. Of the 1,578 detected individual lipid species that were subjected to statistical analysis, TG (4:0/18:2/18:2), CL (23:0/18:1/20:4/20:4), TG (18:0p/18:1/18:2), CL (22:6/18:1/20:0/22:6), LPC (19:3), PC (16:1/16:1), CL (18:2/18:0/18:0/22:0), and TG (20:1/18:2/24:1) were the most critical individual lipids dysregulated by I/R injury in NASH livers (Figure 3).Of the 26 detected lipid classes, SL and CL were identified as the most critical lipid classes being altered in NASH livers with I/R injury (Figure 4). Moreover, according to the known functions of SL and CL in regulating cell death and oxidative stress (27,33-35), the alteration of SL and CL were found to potentially mediate the I/R-induced cell death and oxidative stress in NASH livers (Figure 7). Altogether, these findings highlighted the roles of CL and SL dysregulation in the aggressive I/R injury in NASH livers.

SL family, including CER, SPH, GSL, and SM, is a group of bioactive lipids that regulate various cell biology (36). Dysregulation of SL metabolism has been found in NASH livers or I/R-injured livers $(6,37)$. Our study demonstrated that GSL and SM were significantly decreased, while CER and SPH were increased to higher levels in NASH livers with I/R injury than those in normal livers with I/R injury (Figure $4 D$ ). In SL family, CER is a critical bioactive lipid that was previously found to be upregulated in NASH livers (9) or I/R-injured normal liver (6). It is known that CER are generated through multiple metabolic pathways in mammals (38). They can be synthesized de novo from dihydrosphingosine and fatty acylCoAs through the action of (dihydro) ceramide synthases encoded by 6 distinct genes (CERS1-6). CER can be directly synthesized from SPH and acyl-CoAs by CERS through a salvage pathway. CER can also be generated from the degradation of complex SL, including GSL and SM. Once generated, CER can be hydrolyzed by ceramidases to produce $\mathrm{SPH}$, and $\mathrm{SPH}$ is further phosphorylated by SPH kinases to form S1P. By dissecting the dysregulation of SL metabolic enzymes at mRNA levels, we found that NASH dramatically enhanced the I/R-induced upregulation of Cers2, Acer2, Sphk1, and Smpd3 in livers (Figure 5C). Smpd3 encodes a sphingomyelinase that catalyzes SM to produce CER, which contributes to the degradation pathway of CER generation (39). And Cers2 encodes a major hepatic CER synthase that mediates the de novo and salvage pathway of CER generation in the liver (28). Acer2 encodes a critical ceramidase that hydrolyzes long-chain CER to produce SPH (40). Therefore, the accumulation of CER in I/R-injured NASH livers might mainly be attributed to the upregulation of Smpd3 and Cers2 that produced CER from SM degradation and from CER synthesis, respectively. Notably, in line with the reported substrate specificity of Smpd3 and Cers2 (28,39), we found that the levels of saturated CER with 20 to 26 carbons of fatty acyl chains were substantially increased, and SM with 


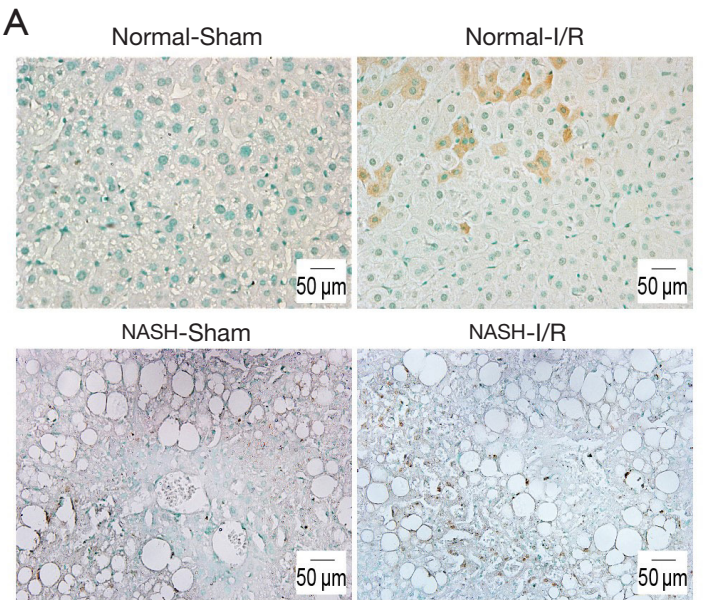

B
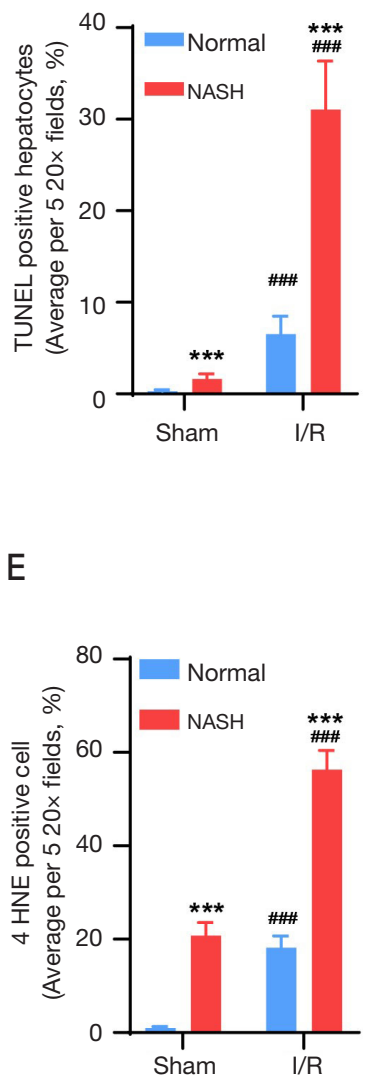

$\mathrm{E}$

$\mathrm{F}$

$\mathrm{F}$
C
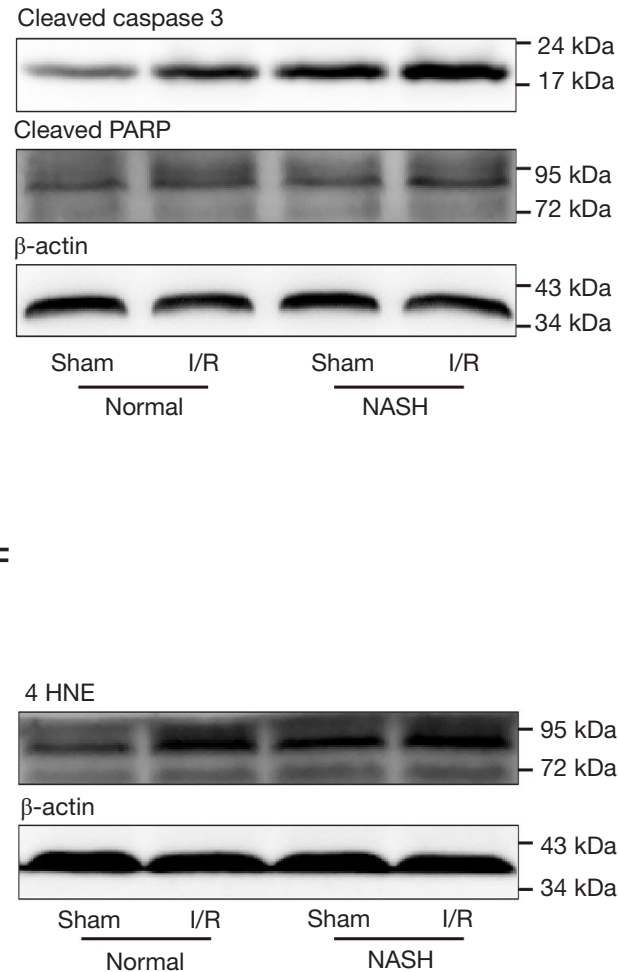

D

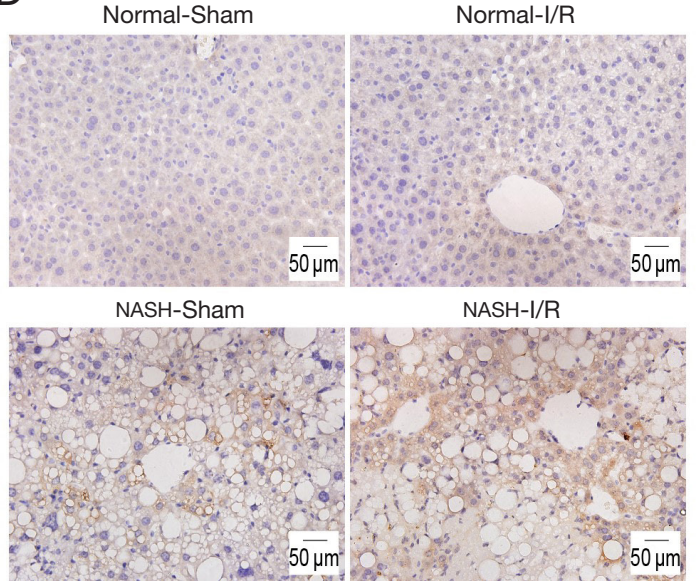

Figure 7 NASH exaggerates I/R-induced cell death and oxidative stress that link to the biofunctions of CER and CL. Mouse livers were collected from normal mice and NASH mice with or without I/R injury (A,B). TUNEL assays were performed to evaluate cell death in livers (A). The positive cells of TUNEL staining were quantified as described in Methods (B). (C) Total proteins were extracted from mouse livers and then subjected to immunoblotting of apoptotic markers, including cleaved-caspase 3 and cleaved-PARP. (D,E) Immunostaining of oxidative stress marker $4 \mathrm{HNE}$ was performed on liver sections (D), and $4 \mathrm{HNE}$ positive cells were quantified as mentioned in Methods (E). (F) Total proteins were extracted from samples of mouse livers and subjected to immunoblotting of 4 HNE. Results were expressed as mean $\pm \mathrm{SD}, \mathrm{n}=6$. ${ }^{* * *}, \mathrm{P}<0.001$, NASH vs. normal; ${ }^{\# \# \#}, \mathrm{P}<0.001, \mathrm{I} / \mathrm{R}$ vs. sham. I/R, ischemia/reperfusion; NASH, nonalcoholic steatohepatitis; TUNEL, transferase dUTP nick end labeling; CER, including ceramides; CL, cardiolipins; SD, standard deviation.

20 to 24 carbons of fatty acyl chains were dramatically decreased in NASH liver with I/R injury (Figure 5D,5E). Finally, the upregulation of Acer2 might catalyze the degradation of the increased CER, thus contributing to the increase of SPH in NASH livers with I/R injury. The mechanism by which I/R injury dysregulated SL metabolism is still unclear. The oxidative stress and TNF- $\alpha$ production during I/R injury have been reported to activate SM hydrolysis and de novo CER synthesis $(41,42)$. These data suggested that both generation and degradation of
CER were highly activated to accelerate the turnover of CER in NASH livers with I/R injury.

CL is a group of major membrane phospholipids in the mitochondria that were previously found to be dysregulated in either NASH or I/R-injured livers (43). CL are synthesized de novo from phosphatidic acid via the cytidine5 -diphosphate-1,2-diacyl-sn-glycerol pathway that is critically catalyzed by CLRS1, and then they are deacylated to monolysocardiolipins to be remodeled into the form that exists in mitochondrial membranes (43). The resynthesis 
of deacylated CL from monolysocardiolipins can be catalyzed by $T a z$ and acyllysocardiolipin acyltransferase-1, monolysocardiolipin acyltransferase- 1 and the alpha subunit of trifunctional protein. Dysregulation of CL metabolism has been previously reported in I/R-injured heart tissues and liver tissues $(17,44)$. Martens et al. reported that $\mathrm{I} / \mathrm{R}$ causes a significant decrease in CL content in the normal liver after 50-min reperfusion (17). Our study demonstrated that after 24-h reperfusion, CL content in NASH livers with I/R injury was significantly decreased, but it was not significantly altered in normal livers with $\mathrm{I} / \mathrm{R}$ injury (Figure 4E). These results indicated that the generation of CL might be suppressed at the early stage of reperfusion then restored in normal livers, but it was persistently impaired in NASH livers with I/R injury. Notably, our enrichment analysis revealed that CL synthesis mediated by Crls1 was indeed activated in normal livers with $\mathrm{I} / \mathrm{R}$ injury. However, the CL synthesis mediated by Crls1 and the CL remodeling catalyzed by Taz were both possibly inhibited in NASH livers with I/R injury (Figure 6E, $6 F$ ). The inactivation of CL generation in NASH livers with I/R injury might be linked to the increases of reactive oxygen species resulting in the oxidation and depletion of mitochondrial CL (45). These data collectively highlighted that the generation of CL might be persistently inactivated to suppress CL production in NASH livers with I/R injury.

Lipotoxicity plays an essential role in mediating hepatic injury in NASH (46). We found that the I/R-induced cell death and oxidative were both exaggerated in NASH livers (Figure 7). Although the conclusively causal role of CER and CL dysregulation in the aggressive I/R injury in NASH livers was not tested in this study, previous studies have reported that accumulation of CER or decrease of CL promoted cell death and oxidative stress related to I/ $\mathrm{R}$ injury. CER were found to directly impair mitochondrial respiration by inhibiting the ubiquinone pool of complex III and nicotinamide adenine dinucleotide phosphate oxidase (47). CER was also known to act as the second messenger that directly induced caspase-related apoptosis (48). On the contrary, CL was known as antioxidative and anti-apoptotic lipids (49,50). A decrease of CL was shown to suppress the respiratory chain in mitochondria resulting in oxidative stress and apoptosis (51). Altogether, these results implicate a potential role of SL and CL in mediating the exaggerated I/R injury in NASH livers.

This study demonstrated the alterations of lipids in NASH liver with I/R injury by untargeted lipidomics, and found that the dysregulation of SL and CL might mediate the exaggerated I/R injury in NASH livers. However, the present study also had some limitations, including (I) all data were generated from the NASH mice, and needed further confirmation in clinical tissues, (II) hepatic lipids alterations were based on untargeted lipidomics approach and warrant further confirmation by targeted lipidomics measurement, (III) it needed further investigation whether the accumulation of CER and decrease of CL could mediate the aggravated hepatic I/R injury in NASH livers through augmenting cell death and oxidative stress, (IV) the model of NASH was established through feeding mice with a saturated-fatty-acid-enriched western-style diet, which might not reflect the human NASH with a more diverse diet, (V) the lipids were assessed at one specific timepoint of I/R injury, and thus it might miss some critical lipids which regulated liver regeneration after I/R injury.

\section{Conclusions}

The I/R-induced dysregulation of CL and SL were critically rewired by $\mathrm{NASH}$, which might potentially mediate the aggressive I/R injury in NASH livers. Therefore, restoring the homeostasis in CL and SL metabolism might have therapeutic potential for hepatic I/R-injury in the NASH liver.

\section{Acknowledgments}

We would like to acknowledge the technical support provided from the Central Laboratory of Southern Medical University, Guangzhou, Guangdong, China for lipidomic analyses.

Funding: This work was supported by the National Natural Science Foundation of China (No. 82070642) to JZ, the Guangdong Province Science and Technology Program (No. 2020A1515011205) to KW, the Guangdong Province Science and Technology Program (No. 2021A1515012146) to CJL, and the President Foundation of Nanfang Hospital, Southern Medical University (No. 2018C029) to JPQ.

\section{Footnote}

Reporting Checklist: The authors have completed the ARRIVE reporting checklist. Available at https://hbsn. amegroups.com/article/view/10.21037/hbsn-21-133/rc

Data Sharing Statement: Available at https://hbsn. 
amegroups.com/article/view/10.21037/hbsn-21-133/dss

Conflicts of Interest: All authors have completed the ICMJE uniform disclosure form (available at https://hbsn. amegroups.com/article/view/10.21037/hbsn-21-133/coif). The authors have no conflicts of interest to declare.

Ethical Statement: The authors are accountable for all aspects of the work in ensuring that questions related to the accuracy or integrity of any part of the work are appropriately investigated and resolved. Experiments were performed under a project license (No. NFYY-2019-028) granted by the institutional ethics committee of Nanfang Hospital Southern Medical University, in compliance with institutional guidelines for the care and use of animals.

Open Access Statement: This is an Open Access article distributed in accordance with the Creative Commons Attribution-NonCommercial-NoDerivs 4.0 International License (CC BY-NC-ND 4.0), which permits the noncommercial replication and distribution of the article with the strict proviso that no changes or edits are made and the original work is properly cited (including links to both the formal publication through the relevant DOI and the license). See: https://creativecommons.org/licenses/by-nc-nd/4.0/.

\section{References}

1. Younossi Z, Anstee QM, Marietti M, et al. Global burden of NAFLD and NASH: trends, predictions, risk factors and prevention. Nat Rev Gastroenterol Hepatol 2018;15:11-20.

2. Tiniakos DG, Vos MB, Brunt EM. Nonalcoholic fatty liver disease: pathology and pathogenesis. Annu Rev Pathol 2010;5:145-71.

3. Cannistrà $M$, Ruggiero $M$, Zullo $\mathrm{A}$, et al. Hepatic ischemia reperfusion injury: A systematic review of literature and the role of current drugs and biomarkers. Int J Surg 2016;33 Suppl 1:S57-70.

4. Wong RJ, Cheung R, Ahmed A. Nonalcoholic steatohepatitis is the most rapidly growing indication for liver transplantation in patients with hepatocellular carcinoma in the U.S. Hepatology 2014;59:2188-95.

5. Losekann A, Weston AC, de Mattos AA, et al. NonAlcoholic Steatohepatitis (NASH): Risk Factors in Morbidly Obese Patients. Int J Mol Sci 2015;16:25552-9.

6. He X, Schuchman EH. Ceramide and Ischemia/ Reperfusion Injury. J Lipids 2018;2018:3646725.
7. Tőkés T, Tuboly E, Varga G, et al. Protective effects of L-alpha-glycerylphosphorylcholine on ischaemiareperfusion-induced inflammatory reactions. Eur J Nutr 2015;54:109-18.

8. Hidalgo FJ, Nogales F, Zamora R. Changes produced in the antioxidative activity of phospholipids as a consequence of their oxidation. J Agric Food Chem 2005;53:659-62.

9. Samuel VT, Shulman GI. Nonalcoholic Fatty Liver Disease, Insulin Resistance, and Ceramides. N Engl J Med 2019;381:1866-9.

10. Pagadala M, Kasumov T, McCullough AJ, et al. Role of ceramides in nonalcoholic fatty liver disease. Trends Endocrinol Metab 2012;23:365-71.

11. Novgorodov SA, Gudz TI. Ceramide and mitochondria in ischemia/reperfusion. J Cardiovasc Pharmacol 2009;53:198-208.

12. Llacuna L, Marí M, Garcia-Ruiz C, et al. Critical role of acidic sphingomyelinase in murine hepatic ischemiareperfusion injury. Hepatology 2006;44:561-72.

13. Wang $\mathrm{K}$, Li C, Lin X, et al. Targeting alkaline ceramidase 3 alleviates the severity of nonalcoholic steatohepatitis by reducing oxidative stress. Cell Death Dis 2020;11:28.

14. Li Q, Qian J, Li Y, et al. Generation of sphingosine-1phosphate by sphingosine kinase 1 protects nonalcoholic fatty liver from ischemia/reperfusion injury through alleviating reactive oxygen species production in hepatocytes. Free Radic Biol Med 2020;159:136-49.

15. Kagan VE, Tyurin VA, Jiang J, et al. Cytochrome c acts as a cardiolipin oxygenase required for release of proapoptotic factors. Nat Chem Biol 2005;1:223-32.

16. Paradies G, Petrosillo G, Pistolese M, et al. Reactive oxygen species affect mitochondrial electron transport complex I activity through oxidative cardiolipin damage. Gene 2002;286:135-41.

17. Martens JC, Keilhoff G, Halangk W, et al. Lipidomic analysis of molecular cardiolipin species in livers exposed to ischemia/reperfusion. Mol Cell Biochem 2015;400:253-63.

18. Matsuzawa N, Takamura T, Kurita S, et al. Lipid-induced oxidative stress causes steatohepatitis in mice fed an atherogenic diet. Hepatology 2007;46:1392-403.

19. Abe Y, Hines IN, Zibari G, et al. Mouse model of liver ischemia and reperfusion injury: method for studying reactive oxygen and nitrogen metabolites in vivo. Free Radic Biol Med 2009;46:1-7.

20. Hong F, Radaeva S, Pan HN, et al. Interleukin 6 alleviates hepatic steatosis and ischemia/reperfusion injury in mice with fatty liver disease. Hepatology 2004;40:933-41. 
21. Goodman ZD. Grading and staging systems for inflammation and fibrosis in chronic liver diseases. J Hepatol 2007;47:598-607.

22. Mehlem A, Hagberg CE, Muhl L, et al. Imaging of neutral lipids by oil red $\mathrm{O}$ for analyzing the metabolic status in health and disease. Nat Protoc 2013;8:1149-54.

23. Yang K, Cheng H, Gross RW, et al. Automated lipid identification and quantification by multidimensional mass spectrometry-based shotgun lipidomics. Anal Chem 2009;81:4356-68.

24. Nguyen A, Rudge SA, Zhang Q, et al. Using lipidomics analysis to determine signalling and metabolic changes in cells. Curr Opin Biotechnol 2017;43:96-103.

25. Arendt BM, Ma DW, Simons B, et al. Nonalcoholic fatty liver disease is associated with lower hepatic and erythrocyte ratios of phosphatidylcholine to phosphatidylethanolamine. Appl Physiol Nutr Metab 2013;38:334-40.

26. Zhai ST, Liu GY, Xue F, et al. Changes of sphingolipids profiles after ischemia-reperfusion injury in the rat liver. Chin Med J (Engl) 2009;122:3025-31.

27. Alonso C, Fernández-Ramos D, Varela-Rey M, et al. Metabolomic Identification of Subtypes of Nonalcoholic Steatohepatitis. Gastroenterology 2017;152:1449-61.e7.

28. Pewzner-Jung Y, Park H, Laviad EL, et al. A critical role for ceramide synthase 2 in liver homeostasis: I. alterations in lipid metabolic pathways. J Biol Chem 2010;285:10902-10.

29. Gonçalves IO, Maciel E, Passos E, et al. Exercise alters liver mitochondria phospholipidomic profile and mitochondrial activity in non-alcoholic steatohepatitis. Int J Biochem Cell Biol 2014;54:163-73.

30. Coelho HR, Berno CR, Falcão GR, et al. Twisting of the spermatic cord: ischemia and reperfusion, toxicogenetic evaluation, and the effects of phosphatidylcholine in preclinical trials. Genet Mol Res 2016;15. doi: 10.4238/ gmr.15038529.

31. Shen XD, Ke B, Zhai Y, et al. Diannexin, a novel annexin $\mathrm{V}$ homodimer, protects rat liver transplants against cold ischemia-reperfusion injury. Am J Transplant 2007;7:2463-71.

32. Zarkovic N. 4-hydroxynonenal as a bioactive marker of pathophysiological processes. Mol Aspects Med 2003;24:281-91.

33. Unal B, Ozcan F, Tuzcu H, et al. Inhibition of neutral sphingomyelinase decreases elevated levels of nitrative and oxidative stress markers in liver ischemia-reperfusion injury. Redox Rep 2017;22:147-59.

34. Ordoñez R, Fernández A, Prieto-Domínguez N, et al. Ceramide metabolism regulates autophagy and apoptotic cell death induced by melatonin in liver cancer cells. J Pineal Res 2015;59:178-89.

35. Liu S, Soong Y, Seshan SV, et al. Novel cardiolipin therapeutic protects endothelial mitochondria during renal ischemia and mitigates microvascular rarefaction, inflammation, and fibrosis. Am J Physiol Renal Physiol 2014;306:F970-80.

36. Hannun YA, Obeid LM. Sphingolipids and their metabolism in physiology and disease. Nat Rev Mol Cell Biol 2018;19:175-91.

37. Régnier M, Polizzi A, Guillou H, et al. Sphingolipid metabolism in non-alcoholic fatty liver diseases. Biochimie 2019;159:9-22.

38. Gault CR, Obeid LM, Hannun YA. An overview of sphingolipid metabolism: from synthesis to breakdown. Adv Exp Med Biol 2010;688:1-23.

39. Chatterjee S. Neutral sphingomyelinase: past, present and future. Chem Phys Lipids 1999;102:79-96.

40. Li F, Xu R, Low BE, et al. Alkaline ceramidase 2 is essential for the homeostasis of plasma sphingoid bases and their phosphates. FASEB J 2018;32:3058-69.

41. Suematsu N, Tsutsui H, Wen J, et al. Oxidative stress mediates tumor necrosis factor-alpha-induced mitochondrial DNA damage and dysfunction in cardiac myocytes. Circulation 2003;107:1418-23.

42. Lecour S, Owira P, Opie LH. Ceramide-induced preconditioning involves reactive oxygen species. Life Sci 2006;78:1702-6.

43. Dudek J. Role of Cardiolipin in Mitochondrial Signaling Pathways. Front Cell Dev Biol 2017;5:90.

44. Paradies G, Paradies V, Ruggiero FM, et al. Mitochondrial bioenergetics and cardiolipin alterations in myocardial ischemia-reperfusion injury: implications for pharmacological cardioprotection. Am J Physiol Heart Circ Physiol 2018;315:H1341-52.

45. Petrosillo G, Portincasa P, Grattagliano I, et al. Mitochondrial dysfunction in rat with nonalcoholic fatty liver Involvement of complex I, reactive oxygen species and cardiolipin. Biochim Biophys Acta 2007;1767:1260-7.

46. Mendez-Sanchez N, Cruz-Ramon VC, Ramirez-Perez OL, et al. New Aspects of Lipotoxicity in Nonalcoholic Steatohepatitis. Int J Mol Sci 2018;19:2034.

47. Holland WL, Summers SA. Sphingolipids, insulin 
resistance, and metabolic disease: new insights from in vivo manipulation of sphingolipid metabolism. Endocr Rev 2008;29:381-402.

48. Gulbins E, Kolesnick R. Acid sphingomyelinase-derived ceramide signaling in apoptosis. Subcell Biochem 2002;36:229-44.

49. Ott M, Zhivotovsky B, Orrenius S. Role of cardiolipin in cytochrome c release from mitochondria. Cell Death

Cite this article as: Yu S, Wang K, Li Q, Wei Y, Li Y, Zhang Q, Huang P, Liang H, Sun H, Peng H, Huang X, Liu C, Zhou J, Qian J, Li C. Nonalcoholic steatohepatitis critically rewires the ischemia/reperfusion-induced dysregulation of cardiolipins and sphingolipids in mice. HepatoBiliary Surg Nutr 2023;12(1):319. doi: 10.21037/hbsn-21-133
Differ 2007;14:1243-7.

50. Paradies G, Paradies V, Ruggiero FM, et al. Oxidative stress, cardiolipin and mitochondrial dysfunction in nonalcoholic fatty liver disease. World J Gastroenterol 2014;20:14205-18.

51. Kagan VE, Bayir HA, Belikova NA, et al. Cytochrome c/ cardiolipin relations in mitochondria: a kiss of death. Free Radic Biol Med 2009;46:1439-53. 
Supplementary

Table S1 The makeup of the control diets for percent calories from protein, carbohydrate and fat (Beijing, China, Beijing Huafukang Biological Technology Co. Ltd, Cat.No.1025)

\begin{tabular}{lcc}
\hline & \% By weight & \% kcal from \\
\hline Protein & $\geq 18.0$ & $\geq 56.2$ \\
Carbohydrate & $\geq 5.0$ & $\geq 15.6$ \\
Fat & $\geq 4.0$ & $\geq 28.1$ \\
\hline
\end{tabular}

Table S2 The makeup of the western diets for percent calories from protein, carbohydrate and fat (TD.88137 diet, Harlan, Southern Easton, MA, USA)

\begin{tabular}{lcr}
\hline & \% By weight & \% kcal from \\
\hline Protein & 17.3 & 15.2 \\
Carbohydrate & 48.5 & 42.7 \\
Fat & 21.2 & 42.0 \\
\hline
\end{tabular}


Table S3 Primers for real-time PCR assays

\begin{tabular}{|c|c|}
\hline Gene & qPCR primer sequence \\
\hline Actb & $\begin{array}{l}\text { 5' -GATGTATGAAGGCTTTGGTC-3' } \\
\text { 5'- TGTGCACTTTTATTGGTCTC-3' }\end{array}$ \\
\hline II-6 & $\begin{array}{l}\text { 5' -AAGAAATGATGGATGCTACC-3' } \\
\text { 5'- GAGTTTCTGTATCTCTCTGAAG-3' }\end{array}$ \\
\hline Tnf-a & $\begin{array}{l}\text { 5'- CATCTTCTCAAAATTCGAGTGACAA-3' } \\
\text { 5'- TGGGAGTAGACAAGGTACAACCC-3' }\end{array}$ \\
\hline Inos & $\begin{array}{l}\text { 5'-AATCTTGGAGCGAGTTGTGG-3' } \\
\text { 5' -CAGGAAGTAGGTGAGGGCTTG-3' }\end{array}$ \\
\hline Cers2 & $\begin{array}{l}\text { 5'-TCATCCCTTCTCAGTATTGGT-3' } \\
\text { 5' -ATCCTTTCGCTTGACATCAG-3' }\end{array}$ \\
\hline Cers4 & $\begin{array}{l}\text { 5'-ACCCTGAATTTGTCCCTGTA-3' } \\
\text { 5' -CTTGAAGTCCTTGCGTTTG-3' }\end{array}$ \\
\hline Cers6 & $\begin{array}{l}\text { 5'-TGTGCCATAGCCCTCAAC-3' } \\
\text { 5' -CTCCGAACATCCCAGTCC-3' }\end{array}$ \\
\hline Spt/c1 & $\begin{array}{l}\text { 5'-TACGAGGCTCCAGCATACC-3' } \\
\text { 5' -TCAGAACGCTCCTGCAACT-3' }\end{array}$ \\
\hline Spt/c2 & $\begin{array}{l}\text { 5'-CCATGCGTCACTGGTTCTA-3', } \\
\text { 5' -GTCCGAGGCTGACCATAAA-3' }\end{array}$ \\
\hline Spt/c3 & $\begin{array}{l}\text { 5'-ACATCCATGAGTCCCGTAG-3' } \\
\text { 5' -TCCATACCTCCAATGTTCC-3' }\end{array}$ \\
\hline Degs1 & $\begin{array}{l}\text { 5'-GGTCGTCCATGAACGAGTA-3' } \\
\text { 5' -TGAAATAGCCAGAGTCCTACAA-3' }\end{array}$ \\
\hline Cerk & $\begin{array}{l}\text { 5'-ATCTCCACGGGACAATAAA-3' } \\
\text { 5' -GGCCATACAGGGCTTTC-3' }\end{array}$ \\
\hline Cerkl & $\begin{array}{l}\text { 5'-GAAGCATGGCTCTTAGGGT-3' } \\
\text { 5' -CTCCTCCTGTGGGCTGTAT-3' }\end{array}$ \\
\hline Asah1 & $\begin{array}{l}\text { 5'-AATAACACTTGGGTTGTCAC-3' } \\
\text { 5' -TAGGATACCCAGATAACCAC-3' }\end{array}$ \\
\hline Asah2 & $\begin{array}{l}\text { 5'-AGAGAGAGCAAGGTATTCTTC-3' } \\
\text { 5' -ACTATTCACAAAGTGGTTGC-3' }\end{array}$ \\
\hline Acer2 & $\begin{array}{l}\text { 5'-GTGTGGCATATTCTCATCTG-3' } \\
\text { 5' -TAAGGGACACCAATAAAAGC-3' }\end{array}$ \\
\hline Acer3 & $\begin{array}{l}\text { 5'-GATTCACTGAGGAACTTTCG-3' } \\
\text { 5' -AGAGAAACTTCACTTTTGGC-3' }\end{array}$ \\
\hline Sphk1 & $\begin{array}{l}\text { 5'-ACAGACCATCCAAAGGTAGTTT-3' } \\
\text { 5' -CTCTATTCTGTGCTCAGTCTGTC-3' }\end{array}$ \\
\hline Sphk2 & $\begin{array}{l}\text { 5'-GTACTCATGTTGGGCATCTT-3' } \\
\text { 5' -CATACTCCACTAACTCCCCA-3' }\end{array}$ \\
\hline Sgp/1 & $\begin{array}{l}\text { 5'-GAACCGACCTCCTCAAGCT-3' } \\
\text { 5' -TCATACACCCAGACTATCAGC-3' }\end{array}$ \\
\hline Sgpp1 & $\begin{array}{l}\text { 5'-GAGCAACTTGCCGCTCTACTA-3' } \\
\text { 5' -GGTCGAGATTCCAGATCCAGAA-3' }\end{array}$ \\
\hline Spd1 & $\begin{array}{l}\text { 5'-GCCTGCAAAGTCTTATTCAC-3' } \\
\text { 5' -CACCACATCGTCCTCAAAG-3' }\end{array}$ \\
\hline Smpd2 & $\begin{array}{l}\text { 5'-GCCCAGTTCATCCACCAC-3' } \\
\text { 5' -CCTCAGTCTCAACGAAAGC-3' }\end{array}$ \\
\hline Smpd3 & $\begin{array}{l}\text { 5'-TCATGGACGTGGCCTATC-3' } \\
\text { 5' -ACCTGCACCTTGAGAAACAG-3' }\end{array}$ \\
\hline Smpd4 & $\begin{array}{l}\text { 5'-GGAATCTCCGATGCCTACA-3' } \\
\text { 5' -ATCATTGGACCACTTGGGT-3' }\end{array}$ \\
\hline Samd8 & $\begin{array}{l}\text { 5'-CAGACCTACCCACCACTCC-3' } \\
\text { 5' -TAGCACAGAATCACGCCAC-3' }\end{array}$ \\
\hline Sgms2 & $\begin{array}{l}\text { 5'-TGGTATTGGTTGGGTTATGG-3' } \\
\text { 5' -CGGGCACAGGTAACGTAGTG-3' }\end{array}$ \\
\hline Galc & $\begin{array}{l}\text { 5'-CCGATTTCCTCTTCCTTGCT-3' } \\
\text { 5' -GGTTCAATATGCGACTCCAA-3' }\end{array}$ \\
\hline Gba2 & $\begin{array}{l}\text { 5'-CGTCCTTTGCCCTCGTC-3' } \\
\text { 5' -TGCCACCACTCCACTCATC-3' }\end{array}$ \\
\hline Gla & $\begin{array}{l}\text { 5'-ACCCTTTCATAAGCCCAATT-3' } \\
\text { 5' -GGTCCAGCGACTTCAACAA-3' }\end{array}$ \\
\hline Enpp7 & $\begin{array}{l}\text { 5'-CGGCAAATACATCGAGAACC-3' } \\
\text { 5' -CTCTGGATACCGAGCGTGGC-3' }\end{array}$ \\
\hline Ugcg & $\begin{array}{l}\text { 5'-GCTTCGTGCTCTTCGTGG-3' } \\
\text { 5' -TTGCCTTCTTGTTGAGGTGT-3' }\end{array}$ \\
\hline
\end{tabular}

